\title{
WS11-E03
}

\section{Gardner Continuation}

\section{S.B. Fomel* (University of Texas at Austin)}

\section{SUMMARY}

I define Gardner continuation as a continuous seismic-data transformation, described by a special linear partial differential equation. Under the usual approximations of prestack time migration, Gardner continuation transforms prestack seismic reflection data to remove both the dependence of moveout velocities on dip and the non-hyperbolic dependence of reflection moveouts on reflector curvature. This provides a useful reversible preconditioner of seismic data for different seismic data processing tasks. 


\section{Amsterdam '14}

\section{Introduction}

The problem of transforming prestack seismic data to zero offset attracted a lot of attention in the geophysical literature, particularly in the 1980s and early 1990s. A number of authors explored the idea of dip moveout, or DMO (Hale, 1995), and the related notions of offset continuation and azimuth moveout. A parallel thread of research focused on improving stacking by exploring the continuity of seismic events across different midpoints using the concepts of multifocusing (Landa et al., 1999) and common-reflection-surface, or CRS (Jäger et al., 2001).

A major flaw in the classic DMO algorithms is related to the very problem that they attempt to address: the dip dependence of the stacking velocity. In order for DMO to perform properly, it must be preceded by normal moveout with dip-independent migration velocity instead of dip-dependent stacking velocity. However, it is not possible to estimate the migration velocity directly from data before DMO is applied. This chicken-and-egg problem was elegantly resolved by Gardner's approach (Gardner et al., 1986; Bednar, 1999), which splits prestack time migration into two consecutive steps: (1) velocity-independent transformation of prestack data and (2) dip-independent velocity analysis.

In this paper, I show that Gardner's transform (step 1 in the process above) can be viewed as a result of a continuous transformation of prestack reflection data, described with the help of a partial differential equation. I call this continuous process Gardner continuation. The continuation proceeds in an auxiliary variable (denoted by $\xi$ ), and the Gardner transform corresponds to continuation from $\xi=0$ to $\xi=1$. Using a theoretical analysis, I prove that, under usual assumptions of prestack time migration, the continuation process removes the dependence of moveout velocities on dip and produces hyperbolic moveouts for reflections from curved reflectors. A numerical implementation of the Gardner continuation enables efficient algorithms for parameter-less reversible transformation of prestack seismic reflection data, which can be used for different data-processing tasks.

\section{Theory}

Let us consider the following partial differential equation:

$$
t \frac{\partial^{2} u}{\partial \xi \partial t}+h \frac{\partial^{2} u}{\partial \xi \partial h}+\frac{h^{2}}{2} \frac{\partial^{2} u}{\partial x^{2}}=0
$$

Equation (1) describes the four-dimensional wavefield $u(x, h, t, \xi)$, where $\{x, h, t\}$ are the coordinates of prestack seismic reflection data ( $x$ is midpoint, $h$ is half-offset, and $t$ is time), and $\xi$ is an auxiliary continuation variable.

Analyzing the geometry of transformaton described by equation (1), we can derive differential equations for transported quantities and solve these equations analytically to obtain

$x_{1}=x_{0}-h_{0} \sin \beta, \quad h_{1}=h_{0} \cos \beta, \quad t_{1}=t_{0} \cos \beta, \quad p_{1}=p_{0} \cos \beta$,

$$
q_{1}=\frac{t_{0} \sin \beta-h_{0} p_{0} \cos ^{2} \beta}{h_{0} \sin \beta} .
$$

Here $x_{0}, h_{0}$, and $t_{0}, p_{0}=\partial T / \partial x$, and $q_{0}=p_{0}=\partial T / \partial h$ correspond to initial conditions for $\mathrm{x}=0$, and

$$
\sin \beta=\frac{p_{0} h_{0}}{t_{0}-q_{0} h_{0}}=\tan \alpha \tan \Theta,
$$

where $\alpha$ is the dip angle and $\Theta$ is the reflection angle (Figure 1). 


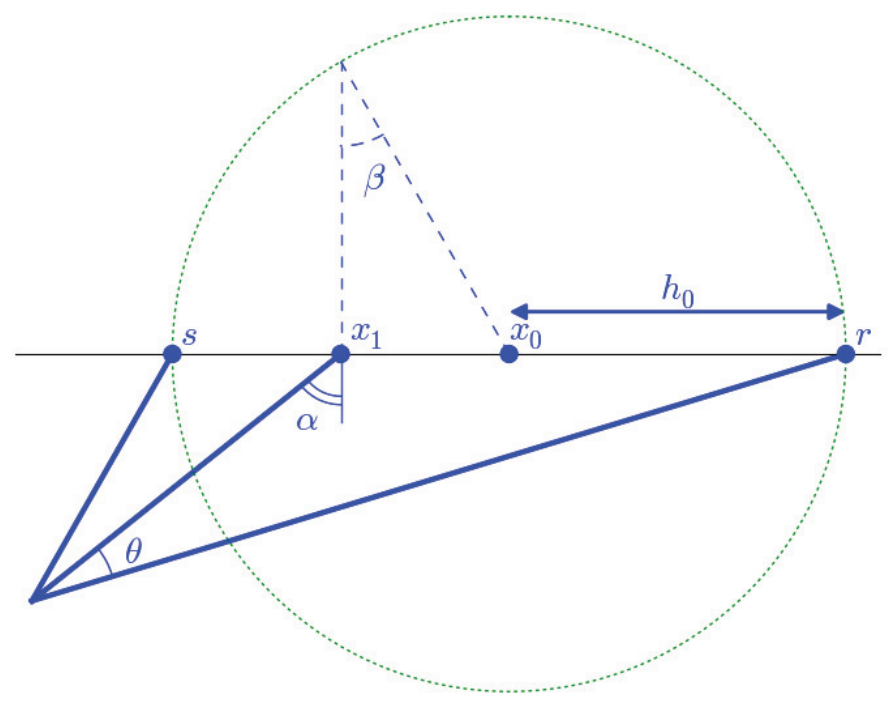

Figure 1 Geometrical relationship between different quantities involved in Gardner continuation and reflection rays (a scheme)

The theory of offset continuation (Fomel, 2003) provides a connection between initial parameters via a partial differential equation. Substituting equations 1-3 into that equation and performing algebraic simplifications leads to the conclusion that, after the Gardner transform (continuation from $\xi=0$ to $\xi=1$ ), the offset-continuation relationship becomes

$$
q_{1}=\frac{4}{v^{2}} \frac{h_{1}}{t_{1}}
$$

and corresponds to the simple hyperbolic moveout with velocity $v$. In other words, reflection moveouts become strictly hyperbolic and dip-independent, as originally indicated by Gardner et al. (1986). Therefore, CRS approximation can be applied directly after the Gardner transform, without the need to account for nonhyperbolicity of diffraction events or reflections from curved reflectors (Landa et al., 2010; Fomel and Kazinnik, 2013). This theory and its conclusions easily extend to 3D.

\section{Conclusions}

Gardner continuation is a continuous process of seismic data transformation. Under the usual assumptions of isotropic prestack time migration, this transformation makes seismic events in CMP gathers strictly hyperbolic and dip-independent. Thanks to this property, continuation theory can be used to design a useful parameterless reversible transformation of prestack seismic data.

\section{References}

Bednar, J. B., 1999, A theoretical comparison of equivalent-offset migration and dip moveout prestack imaging: Geophysics, 64, 191-196.

Fomel, S., 2003, Theory of differential offset continuation: Geophysics, 68, 718-732.

Fomel, S., and R. Kazinnik, 2013, Nonhyperbolic common reflection surface: Geophysical Prospecting, 61, 21-27.

Gardner, G. H. F., S. Y. Wang, N. D. Pan, and Z. Zhang, 1986, Dip moveout and prestack imaging: 18th Annual Offshore Technology Conference, OTC 5158.

Hale, D., ed., 1995, DMO processing: Society of Exploration Geophysicists

Jäger, R., J. Mann, G. Hocht, and P. Hubral, 2001, Common-reflection-surface stack: Image and attributes: Geophysics, 66, 97-109.

Landa, E., B. Gurevich, S. Keydar, and P. Trachtman, 1999, Application of multifocusing method for subsurface imaging: Journal of Applied Geophysics, 42, 283-300.

Landa, E., S. Keydar, and T. J. Moser, 2010, Multifocusing revisited - Inhomogeneous media and curved interfaces: Geophysical Prospecting, 58, 925-938. 